\title{
Denys DUTYKH
}

CNRS-LAMA, University of Savoie, France

Elena TOBISCH

Johannes Kepler University, Linz, Austria

\section{DIRECT DYNAMICAL ENERGY CASCADE IN THE MODIFIED KDV EQUATION}




\title{
DIRECT DYNAMICAL ENERGY CASCADE IN THE MODIFIED KDV EQUATION
}

\author{
Denys Dutykh And Elena ToBisch*
}

\begin{abstract}
In this study we examine the energy transfer mechanism during the nonlinear stage of the Modulational Instability (MI) in the modified Korteweg-de Vries (mKdV) equation. The particularity of this study consists in considering the problem essentially in the Fourier space. A dynamical energy cascade model of this process originally proposed for the focusing NLS-type equations is transposed to the mKdV setting using the existing connections between the KdV-type and NLS-type equations. The main predictions of the $D$-cascade model are outlined and validated by direct numerical simulations of the $\mathrm{mKdV}$ equation using the pseudo-spectral methods. The nonlinear stages of the MI evolution are also investigated for the $\mathrm{mKdV}$ equation.
\end{abstract}

Key words and phrases: Modulational Instability; energy cascade; Korteweg-de Vries equation; modified KdV equation; NLS equation.

MSC: [2010]35Q53 (primary), 35Q55, 76E30 (secondary)

\footnotetext{
* Corresponding author.
} 


\section{Contents}

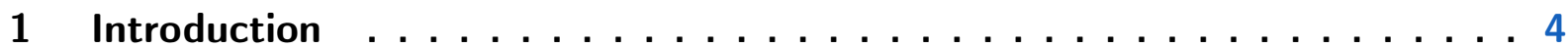

$2 \quad D$-cascade in the model equation $\ldots \ldots \ldots \ldots \ldots$

3 Numerical simulations . . . . . . . . . . . . . . . . 7

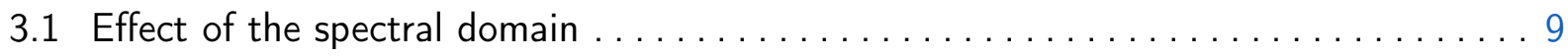

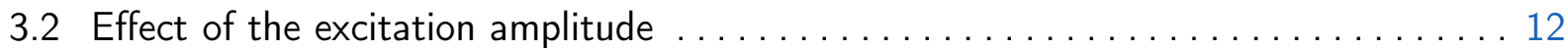

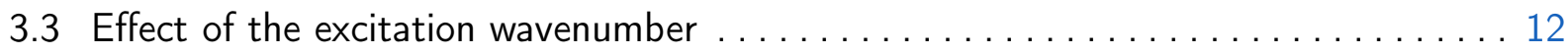

3.4 Effect of the perturbation magnitude $\ldots \ldots \ldots \ldots \ldots \ldots \ldots \ldots \ldots \ldots \ldots \ldots \ldots \ldots \ldots$

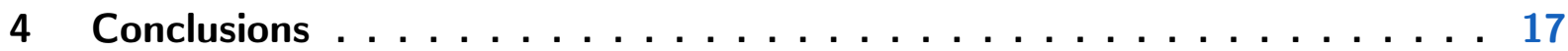

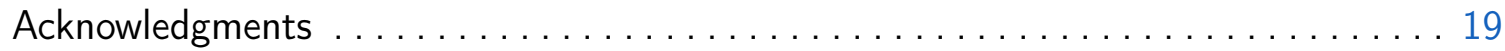

References $\ldots \ldots \ldots \ldots \ldots \ldots$ 


\section{Introduction}

Nonlinear wave systems occur in numerous physical areas from optics to fluid mechanics, from astronomy to geophysics, and one of the most important issues regarding these systems is a description of its energy behavior. One of the most beautiful examples to illustrate this point, is the hypothesis of Kolmogorov on the form of energy spectrum in systems with strong turbulence in which the energy spectrum is supposed to have the universal form $\mathcal{E}(\ell) \sim \ell^{-5 / 3}$ where $\ell$ is the size of the eddy, [25]. In the kinetic weak (or wave) turbulence theory (WTT) dispersive waves play now the role of eddies, and the energy spectrum is again power law $k^{-\alpha}$ where $k$ is the wave length (if dispersion function $\omega \sim k^{\beta}$ ) and $\alpha$ is not universal any more, [37].

The kinetic WTT is an asymptotic theory which is working for very small nonlinearity $0<\varepsilon \lesssim 0.01$, where small parameter $\varepsilon$ is usually taken as a product of wave amplitude with wave number, $\varepsilon=A k$. The smallness of $\varepsilon$ is very important while the kinetic WTT is essentially based on the following assumption: time scales for $3-, 4-, \ldots, s$-wave resonances are separated and can be studied independently. This assumption breaks at about $\varepsilon \approx 0.1$, e.g. [2]. On the other hand, usual laboratory experiments and numerical simulations are performed for $\varepsilon \approx 0.1 \div 0.4$ while for a smaller $\varepsilon \sim 0.01$ corresponding time scales are too long and kinetic energy cascades can not be observed in an experiment at the present stage of technical facilities, [24, 27].

A new model (hereafter referred to as $D$-model) for the formation of the energy spectrum has been developed by E. KARTASHOVA (2012) in [22]; the model can be applied for describing nonlinear wave systems with nonlinearity parameter of the order of $\varepsilon \sim 0.1 \div$ 0.4 and wave systems with narrow frequency band excitation. Basic physical mechanism responsible for the formation of the energy spectrum in this model is not a common $s^{-}$ wave resonance but the modulation instability, and the main assumption of the model is that energy cascade is formed by the most unstable modes in the system, i.e. modes with maximum increment of instability. In [22], the Increment Chain Equation Method (ICEM) was developed for computing dynamical energy spectrum in the systems possessing modulation instability, and applied for the focusing NLS and mNLS, with different levels on nonlinearity, [23, 31].

The NLS is a very attractive equation because of its integrability, but unfortunately it gives sometimes not good enough description of the observed physical effects. For instance, modulation instability was discovered in laboratory experiments with water waves and explained by BENJAMIN \& FEIR (1967) [3], as instability of a narrow wave packet in the framework of the NLS. However, numerical simulations with NLS demonstrate a symmetric energy cascade in the Fourier space while energy cascade experimentally observed in a water tank, is asymmetric. To cope with this problem, it is necessary to introduce various modifications to NLS, e.g. [10, 19]. These modifications allow for the realistic values of small parameter, $0<\varepsilon_{\text {real }} \sim 0.1 \div 0.4$, and are more suitable for modeling real physical phenomena. 
The Korteweg-de Vries (KdV) equation along with its various modifications is another widely used model equation describing long waves, i.e. the region of small wave vectors $k d \ll 1, d$ being the mean water depth. This equation does not have the restrictive assumption of narrow spectrum as the NLS equation. However, many modifications of $\mathrm{KdV}$ are integrable which is a strong mathematical property. So it is not surprising that the KdV equation has found many applications in different fields of Physics such as the shallow water wave dynamics [8,36], internal waves in two-component fluids [15] and acoustic waves in plasmas [30]. Our motivation to study the KdV-family of equations comes mainly from the numerous real-world applications that it can cover.

Though KdV does not have modulation instability [1], its various modifications do possess this property, under certain conditions. Thus, perturbations of a quasi-periodical wave train with small amplitudes in the generalized $\mathrm{KdV}$ equations with nonlinearity of the form $\left(u^{p+1}\right)_{x}$

$$
\operatorname{gKdV}\left(u_{ \pm}\right) \doteq u_{t}+u_{x x x}+\left(u^{p+1}\right)_{x}=0
$$

are modulationally stable if $p<2$, while they are modulationally unstable if $p>2$, [18]. A more general version of this result allowing for nonlocal dispersion can be found in [21]. However, all these results do not allow to obtain a nice analytical representation for the instability interval as in [3], and a numerical study is unavoidable.

Another reference point important for our study of $\mathrm{mKdV}$ is the following remarkable feature of this equation: it can be reduced, under certain conditions, to the mNLS where the MI can be studied by analytically. This reduction can be made by the variational methods [34] or by standard asymptotical approach as in [15].

Our aim in this paper is to study a particular case with $p=2$ and one space dimension — the so-called modified Korteweg-de Vries (mKdV) equation

$$
\operatorname{mKdV}\left(u_{ \pm}\right) \doteq u_{t}+u_{x x x} \pm 6 u^{2} u_{x}=0
$$

with $u$ being a real-valued scalar function, $x$ and $t$ are space and time variables consequently, and the subscripts denote the corresponding partial derivatives. As a starting point for our simulations aiming to study the MI in the $\mathrm{mKdV}$, i.e. $p=2$ in (1.1), we use the estimates obtained in [18] by combination of analytical results and numerical estimates, namely that for $p=2$, the wave is spectrally stable for all wave vectors $0<k^{2}<2$.

It is also shown in [15] that wave packets are unstable only for a positive sign of the coefficient of the cubic nonlinear term in (1.2), and for a high carrier frequency. Being interested in modulation instability, we restrict the study further on the case of focusing mKdV equation:

$$
\operatorname{mKdV}\left(u_{+}\right) \doteq u_{t}+u_{x x x}+6 u^{2} u_{x}=0 .
$$

In the present paper we aim to study in detail formation and properties of the direct $D$-cascade in the frame of $\mathrm{KdV}$ equation (1.3).

The present manuscript is organized as follows. In Section 2 we give a sketch of a $D$-cascade formation for this equation and formulate the properties of the cascade and its spectra which should be verified numerically. In Section 3 we describe shortly our numerical 
approach and present results of our numerical simulations. Finally, the main conclusions of this study are briefly formulated in Section 4.

\section{D-cascade in the model equation}

The main effect of the Modulational Instability (MI) is the disintegration of periodic wavetrains into side bands. BENJAMIN \& FEIR (1967) [3] showed that there is a connection between the frequencies, wavenumber and amplitudes of unstable modes in the framework of the focusing $(+)$ Nonlinear Schrödinger (NLS) equation, which reads after a proper re-scaling:

$$
\operatorname{NLS}\left(v_{ \pm}\right) \doteq \mathrm{i} v_{t}+v_{x x} \pm|v|^{2} v=0
$$

Namely, they computed the instability interval in the form

$$
0<\Delta \omega / A k \omega \leq \sqrt{2},
$$

where $\omega(k)$ is the linear dispersion relation, $k$ is the wavenumber and $A$ is the amplitude of the Fourier mode $\omega$. Quantity $\Delta \omega$ is the distance between the parent mode and its side band. It was also shown in [3] that the most unstable mode satisfies the following relation:

$$
\Delta \omega / A k \omega=1 \text {. }
$$

The use of two assumptions - a) an energy cascade is formed by the most unstable modes, and b) the energy fraction $p$ (called cascade intensity) transported from one cascading mode to the next one is constant, allows to construct and to solve an approximate ordinary differential equation for computing amplitudes of cascading modes, [22]. The first constitutive assumption was inspired by the well-known hypothesis of O. Phillips while the second - by numerous experimental studies of water waves, e.g. [35].

The amplitude of the $n$-s mode in the cascade can be computed as

$$
A\left(\omega_{ \pm n}\right)= \pm(\sqrt{p}-1) \int_{\omega_{0}}^{\omega_{+n}} \frac{\mathrm{d} \omega_{n}}{\omega_{n} k_{n}}+C_{ \pm}\left(\omega_{0}, A_{0}, p\right)
$$

Accordingly, by definition the energy $E_{n}\left(\omega_{n}\right) \propto A^{2}\left(\omega_{n}\right)$, which provides us with the discrete set of energies of individual harmonics. The spectral density $\mathcal{E}^{(\mathrm{Dir})}(\omega)$ can be now computed

$$
\mathcal{E}^{(\operatorname{Dir})}(\omega) \doteq \lim _{\Delta \omega_{n} \rightarrow 0} \frac{E\left(\omega_{n+1}\right)-E\left(\omega_{n}\right)}{\Delta \omega_{n}} .
$$

A similar formula can be written for the inverse cascade as well. However, the limits of integration in (2.3) will be inverted correspondingly to $\int_{\omega_{-n}}^{\omega_{0}}$.

Besides the form of energy spectra, the $D$-cascade model allows to make other predictions for the Nonlinear Schrödinger (NLS)-family of the PDEs e.g.. the time scale for the $D$ cascade occurring is $t_{\mathrm{MI}} \propto t / \varepsilon^{2}$; the distance between two cascading frequencies depends on the steepness of the initial wave train; the cascade can be determined, depending on the choice of excitation parameters $\omega_{0}, k_{0}, A_{0}$; the $D$-cascade termination can be caused by a few main reasons: stabilization, wave breaking and intermittency. All scenarios were observed experimentally, e.g. in [7,33]. 
Connection between the Nonlinear Schrödinger (NLS) and the mKdV can be established in the following way. As it was shown by R. GRIMSHAW (2002) [14], the following generalized Korteweg-de Vries ( $\mathrm{gKdV}$ ) equation

$$
\operatorname{gKdV}: v_{t}+v v_{x}+v^{2} v_{x}+v_{x x x}=0
$$

can be regarded as weakly nonlinear model for internal long waves in a stratified fluid. This model equation can be reduced to the $\mathrm{mKdV}$ equation (1.2) with positive nonlinearity:

$$
u_{t}+u_{x x x}+6 u^{2} u_{x}=0
$$

which in turn the classical Nonlinear Schrödinger (NLS) equation to an asymptotic accuracy $\mathcal{O}\left(\varepsilon^{4}\right)$ :

$$
\mathrm{i} \frac{\partial A}{\partial \tau}=3 k \frac{\partial^{2} A}{\partial \xi^{2}}+6 k|A|^{2} A
$$

after suitable change of variables.

By derivation equation (2.4) describes the envelope propagation for the mKdV solutions. Since both models are modulationally unstable, the $D$-cascade model can be applied to them.

However, the NLS equation is built upon the additional assumption of the narrow band spectrum. Consequently, the $D$-cascade for a single NLS equation consists of only one cascading mode, possibly accompanied with the spectrum broadening. This situation is illustrated on Figure 1 where equation (2.4) was solved numerically for a modulated plane wave initial condition.

The full description of a non-trivial cascade in the NLS framework requires a sequence of the NLS models: each NLS equation describing the vicinity of a cascading mode. On the other hand, the mKdV equation does not have such restrictions. Consequently, we can expect to observe the full $D$-cascade in this model. Numerical study of the inverse $D$ cascade in the $\mathrm{mKdV}$ equation is given in [9], while below we focus on the direct $D$-cascade formation in the same equation.

\section{Numerical simulations}

In order to solve numerically the $\mathrm{KdV}$ equation on a periodic domain we used the classical Fourier-type pseudo-spectral method [4, 32]. The derivatives are computed in the Fourier space, while the nonlinear products - in the physical one (with the linear CPU-time). Thanks to the FFT algorithm [11-13] the passage between these two representations is done in the super-linear time $\mathcal{O}(N \log (N))$, which determines the overall algorithm complexity (per time step). For the dealiasing we used the classical 2/3-rule [32] which was combined (when necessary) with the Fourier smoothing method proposed in [20] for more delicate treatment of higher frequencies. The discretization in time was done with the embedded adaptive $5^{\text {th }}$ order Cash-Karp Runge-Kutta scheme [6] with the adaptive PI step size control [17]. 

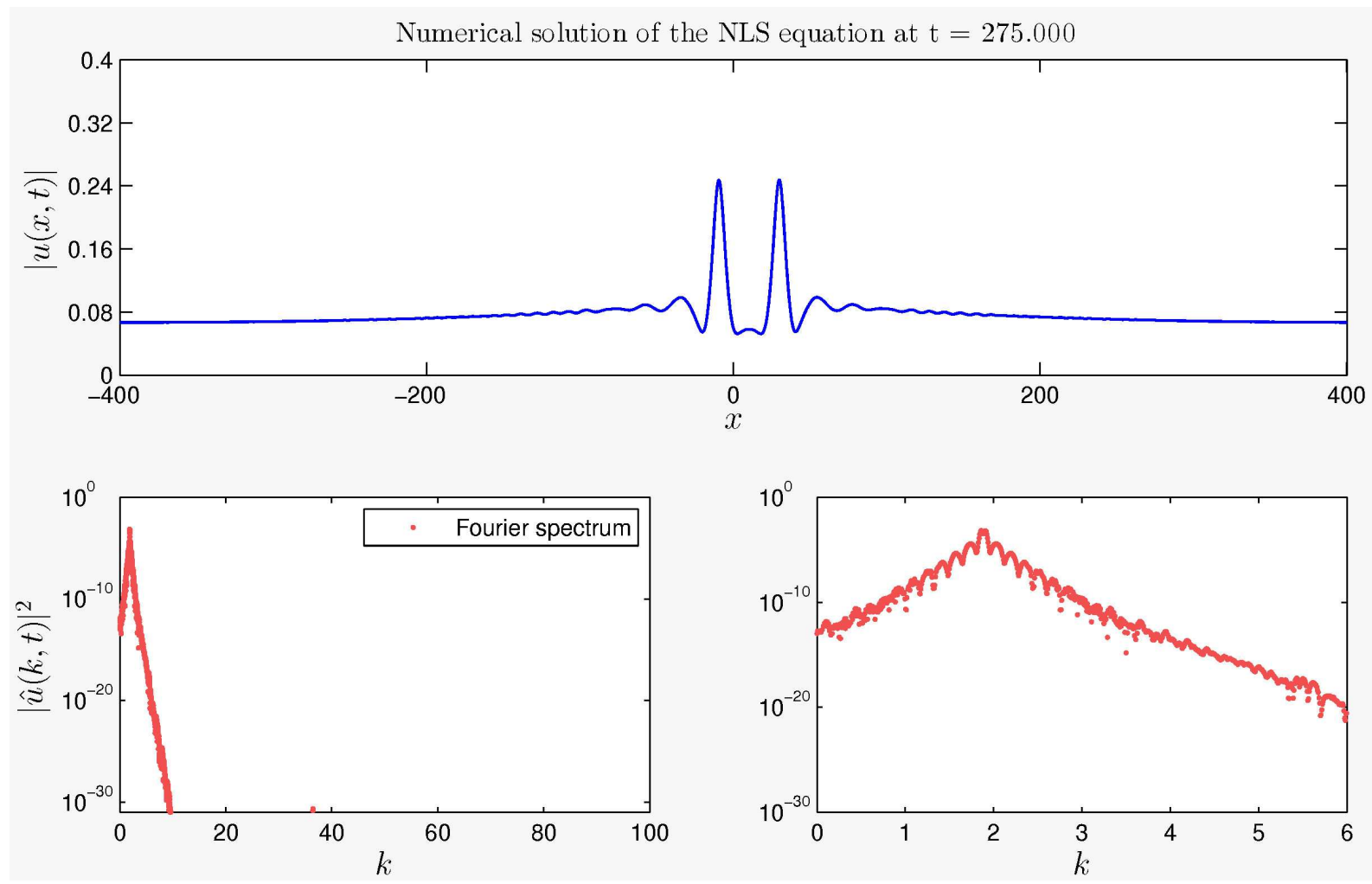

Figure 1. MI in the NLS equation (2.4) for the initial condition parameters given in Table 1.

As there are several exact analytical solutions known for the (1.3) equation, we use them for the verifying our numerical model. The exact cnoidal wave solutions to the KdV equation can be found in [5]:

$u(x, t)=\frac{1}{\frac{s}{3 s^{2}-1}+\frac{\sqrt{2\left(1-s^{2}\right)}}{6 s^{2}-2} \sin \left(\sqrt{3 s^{2}-1}\left(x-x_{0}-t\right)\right)}-s, \quad s \in\left[-1,-\frac{\sqrt{3}}{3}\right] \cup\left[\frac{\sqrt{3}}{3}, 1\right], \quad x_{0} \in \mathbb{R}$.

The one-soliton solution is given by

$$
u(x, t)=a+\frac{b^{2}}{\sqrt{4 a^{2}+b^{2}} \cosh y+2 a}, \quad y=b x-\left(6 a^{2} b+b^{3}\right) t+c,
$$

where $a, b, c$ are arbitrary constants, [26]. There exists also a rational solution of the form

$$
u(x, t)=a-\frac{4 a}{4 a^{2}\left(x-6 a^{2} t\right)^{2}+1},
$$

where $a$ is arbitrary constant, [28].

The numerical solver was validated first on the simple tests of the cnoidal wave propagation and the overtaking collision of solitary waves. We checked that up to the numerical accuracy the spectrum was stationary in the former simulation showing that the wave is 


\begin{tabular}{|l||c|}
\hline \hline Base wave amplitude, $a$ & 0.08 \\
Perturbation magnitude, $\delta$ & 0.05 \\
Base wavenumber, $k_{0}$ & 1.884 \\
Perturbation wavenumber, $K_{0}$ & 0.00785 \\
Ratio of wavelengths, $k_{0} / K_{0}$ & 240 \\
\hline \hline
\end{tabular}

Table 1. Numerical parameters from the previous study [16] used for the comparison with the present results.

perfectly preserved by the numerical solver and no dispersive tail was present after the collision in the latter, confirming the integrability of the $\mathrm{mKdV}$ equation.

For our numerical simulations of the Modulational Instability (MI) we adopt the set-up used also earlier in [16]. We consider the following initial condition posed on a periodic domain $[-\ell, \ell]=\left[-\pi / k_{0}, \pi / k_{0}\right]$ :

$$
u(x, 0) \equiv u_{0}(x)=a\left(1+\delta \sin \left(K_{0} x\right)\right) \sin \left(k_{0} x\right),
$$

where $a$ is the base wave amplitude, $\delta$ is the perturbation magnitude and the wavenumbers $k_{0}, K_{0}$ are chosen such that their ratio $k_{0} / K_{0} \in \mathbb{Z}$. The values of parameters are given in the Table 1. The number $N$ of Fourier modes used in simulations presented below will be set to $N=32768$ and the tolerance parameter in the PI step size control was set to $10^{-8}$.

\subsection{Effect of the spectral domain}

For the Fourier domain limited to $k \in[0,8]$ the simulation results shown on Figures 2 and 3 are in a good qualitative agreement with the results presented in [16]. Unfortunately the authors in [16] did not report the evolution of the Fourier spectrum. We will fill in this gap in the present study. For instance, one can notice the presence of a second peak in the Fourier spectrum (in the vicinity of $k \approx 6$ ) which corresponds to the second cascading mode. During the development of the MI we observe a broadening of the spectrum around two cascading modes present in this numerical simulation. This broadening continues until the MI is fully developed (see Figure $3(c, d)$ ). The left and right broadening wings can appear to be symmetric up to the graphical resolution, however our measurements reported in Table 2 show that it is not actually the case. The broadening intensities defined as the ratio of energies of two consecutive modes, i.e. $\beta_{\mathrm{r}, 1} \doteq \frac{\left|u_{k \pm 1}\right|^{2}}{\left|u_{k}\right|^{2}}$ and observed in the experiments depend on the initial wave amplitude. Namely, when the nonlinearity is increased, the broadening tails become flatter, thus involving more Fourier harmonics into this process.

In order to observe more than two cascading modes, the Fourier space has to be broaden. These results are shown on the left panel of Figure 4. In this case the Fourier domain is almost four times larger going up to $k=32$. Accordingly to the theoretical predictions, 


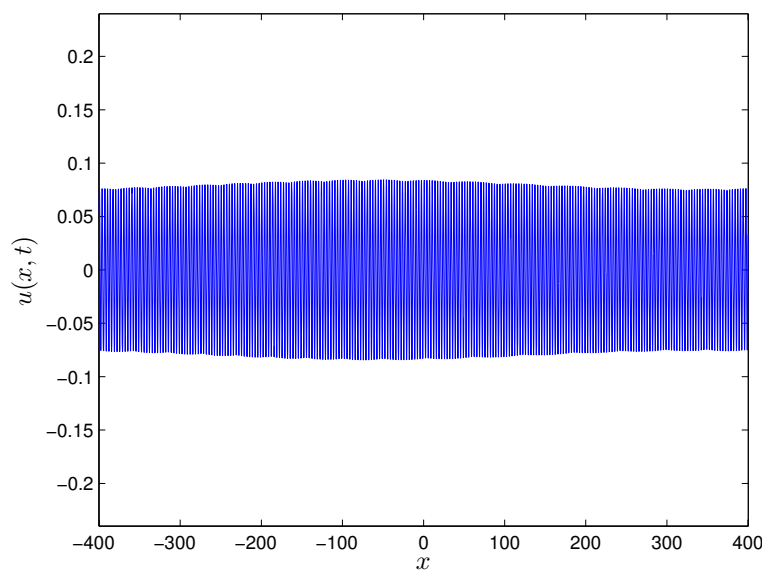

(a) Solution at $t=100.0$

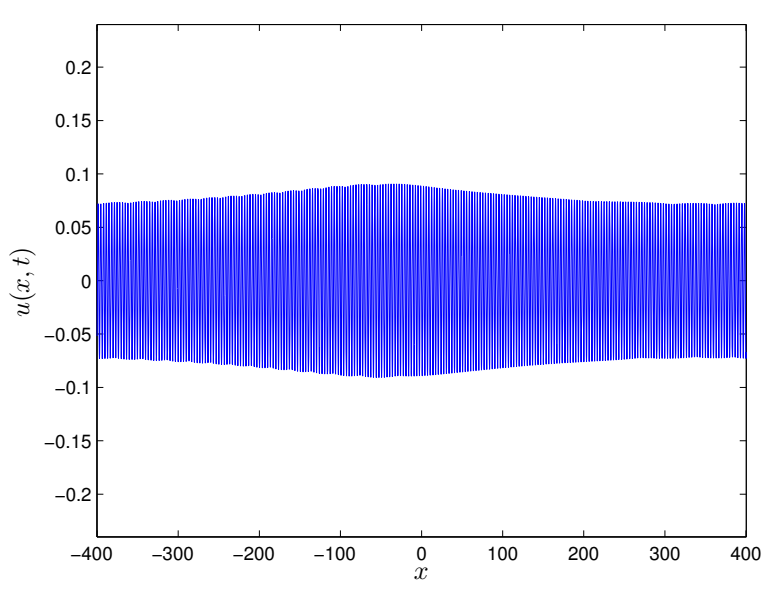

(c) Solution at $t=400.0$

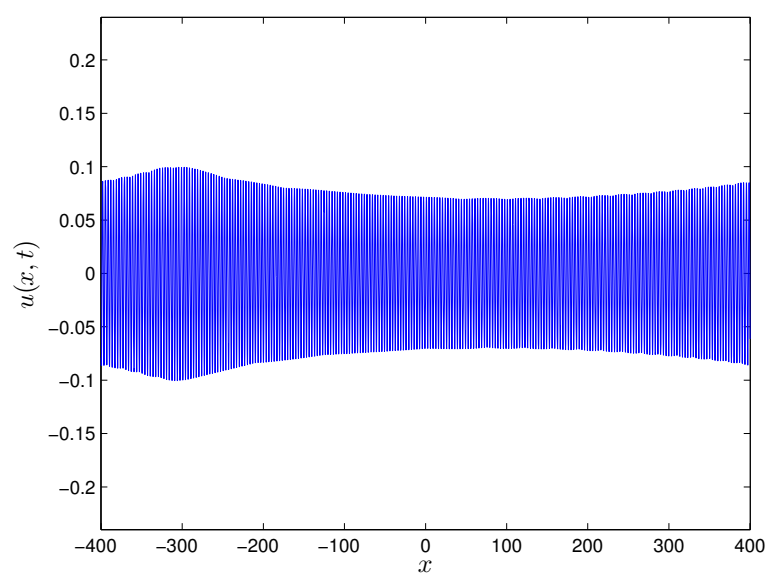

(e) Solution at $t=510.0$

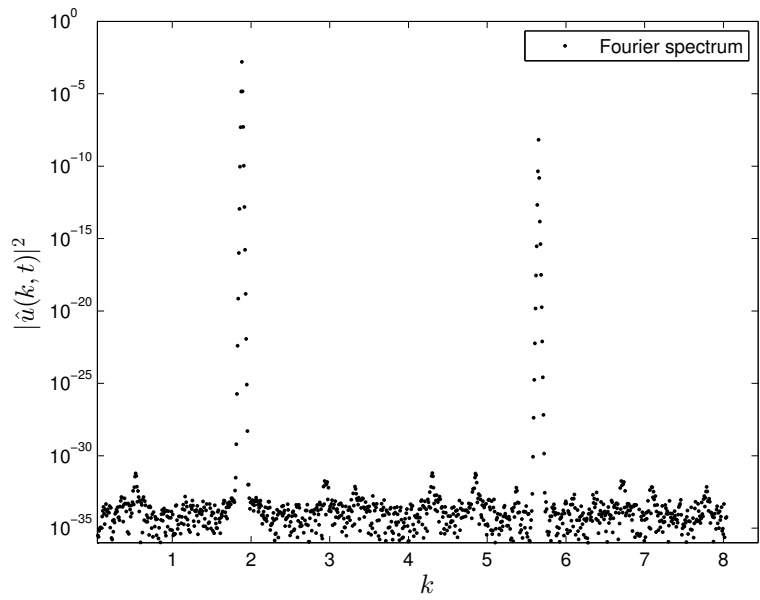

(b) Fourier spectrum at $t=100.0$

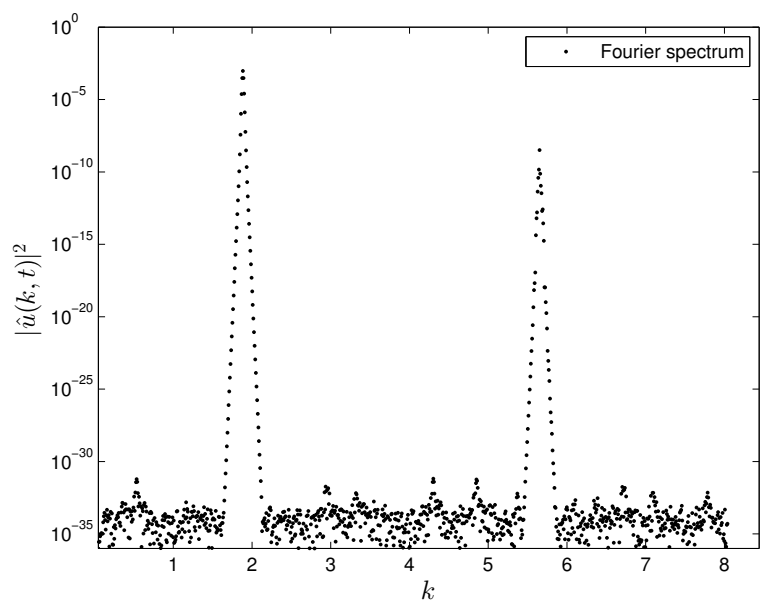

(d) Fourier spectrum at $t=400.0$

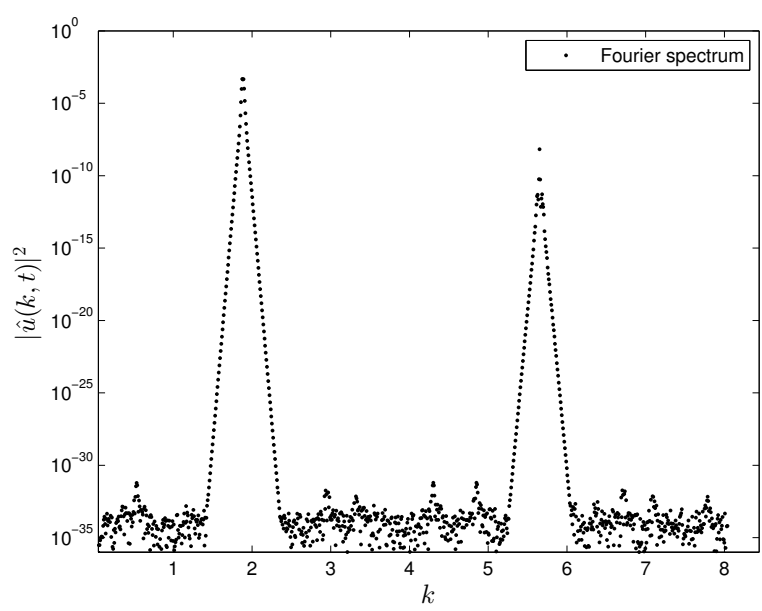

(f) Fourier spectrum at $t=510.0$

Figure 2. Development of the modulational instability in the $m K d V$ equation for parameters given in Table 1. The left panel shows the $m K d V$ solution and on the right panel we show the Fourier spectrum for $k \in[0,8]$. 


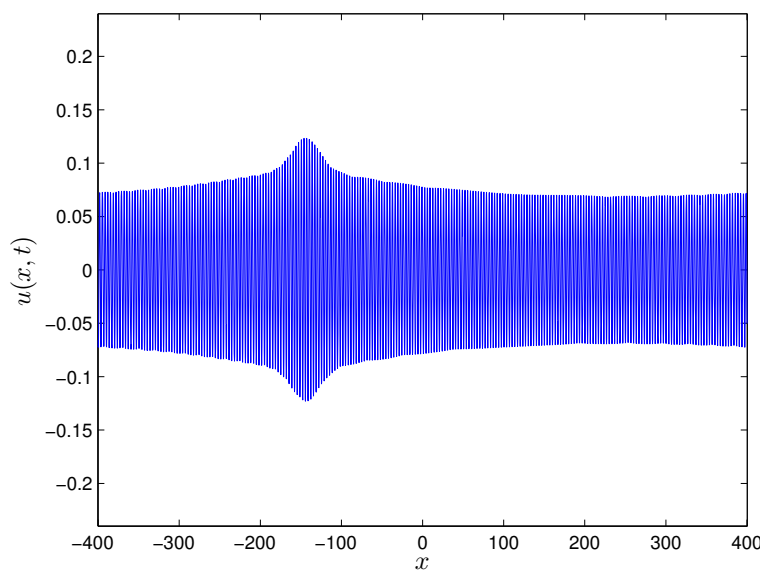

(a) Solution at $t=560.0$

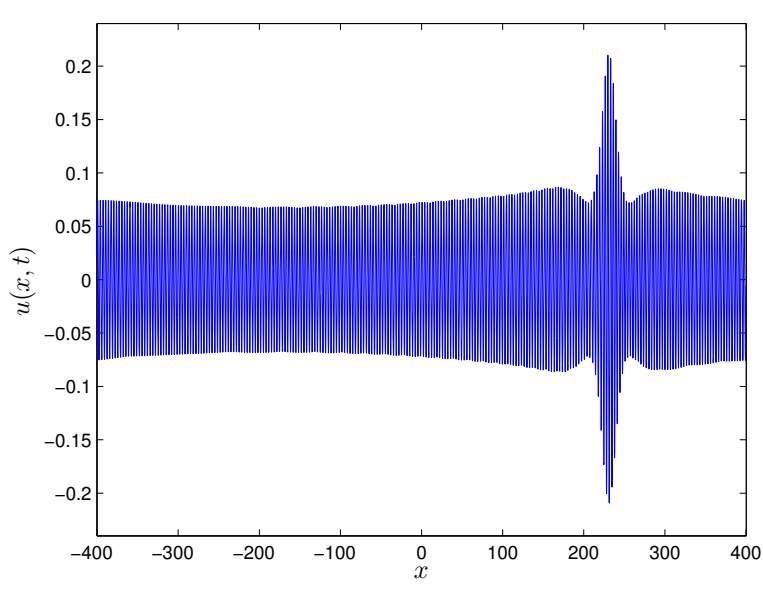

(c) Solution at $t=600.0$

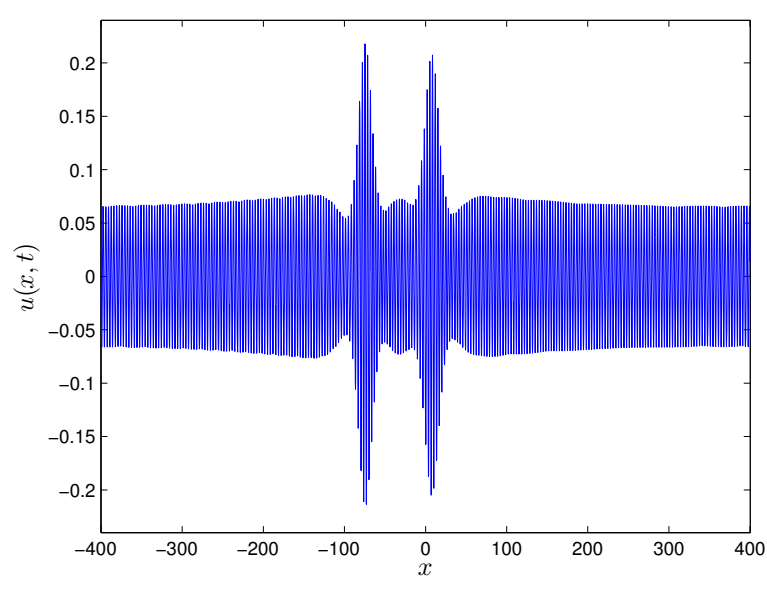

(e) Solution at $t=700.0$

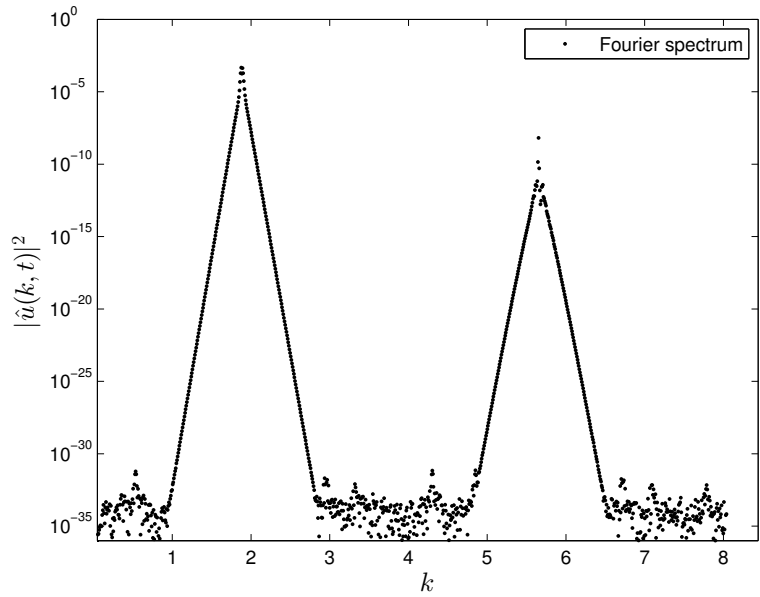

(b) Fourier spectrum at $t=560.0$

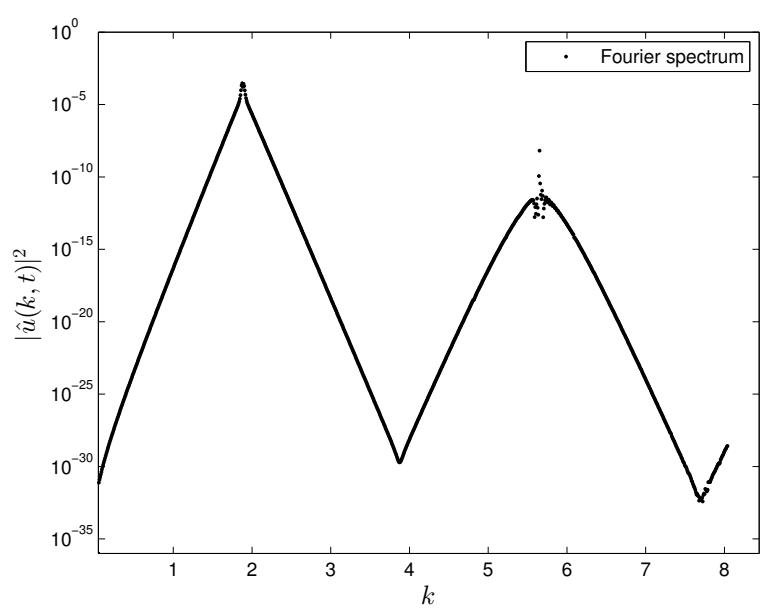

(d) Fourier spectrum at $t=600.0$

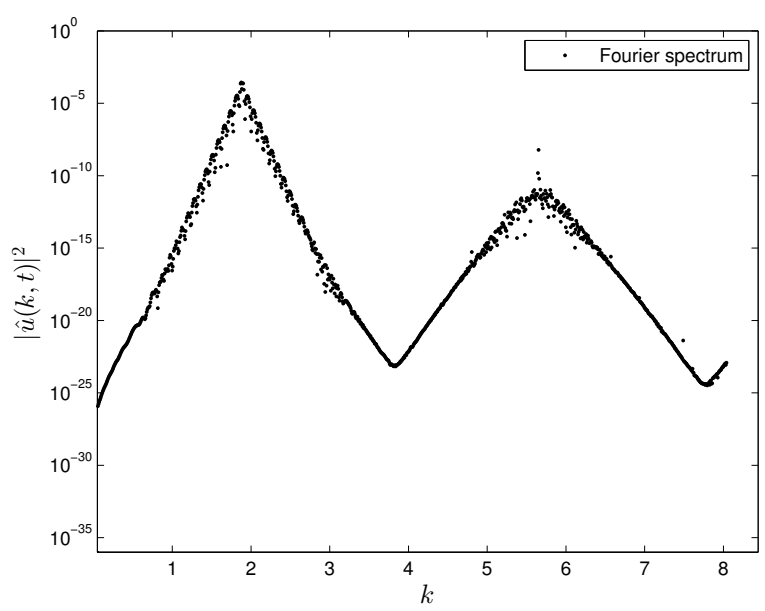

(f) Fourier spectrum at $t=700.0$

Figure 3. (Continued). See Figure 2 for the detailed description. 


\begin{tabular}{|c||c|c|}
\hline \hline Amplitude, $a$ & Left intensity, $\beta_{l}$ & Right intensity, $\beta_{r}$ \\
\hline \hline 0.02 & 2.109 & 2.085 \\
0.03 & 1.590 & 1.550 \\
0.04 & 1.406 & 1.367 \\
0.05 & 1.340 & 1.314 \\
0.08 & 1.292 & 1.253 \\
\hline \hline
\end{tabular}

Table 2. The left and right spectrum broadening intensities measured in numerical simulations of the $m K d V$ equation for different values of the base wave amplitude. The measurements were made at the fully developed MI.

the direct $D$-cascade is formed from the first instances of the numerical simulation. The linear fit in the Fourier space suggests the exponential shape of the energy spectrum $\mathcal{E}_{k} \propto$ $\exp (-\alpha k)$, for some positive value of the slope $\alpha$. Moreover, we computed the distances between two successive cascading modes. It turns out that this value is constant to the numerical precision (obviously, for a fixed initial condition).

\subsection{Effect of the excitation amplitude}

In this Section we study the effect of the base wave amplitude on the development of the MI in the mKdV equation. Namely, we perform the same simulation described in the previous section with numerical parameters given in Table 1, except for the amplitude $a$ which will be chosen as $2 a=0.16$ and $3 a=0.24$. Moreover, we will take a larger spectral domain $k \in[0,32]$ in contrast to the previous case. A larger domain is precisely needed to observe several cascading modes and to make some conclusions on their distribution in the Fourier domain. The simulation results are shown on Figures 4 and 5 .

In particular, one can see that the initial condition with the amplitude two times bigger ( $a=0.16$ ) develops the MI much faster. According to the theoretical predictions [24], the time $T_{\mathrm{MI}}$ needed for the MI to be fully developed in the physical space scales as $T_{\mathrm{MI}} \propto \mathcal{O}\left(\varepsilon^{-2}\right)$. So, the increase of the amplitude leads also to the increase in the nonlinearity $\varepsilon$. Consequently, our numerical results corroborate this prediction of the theory. On the other hand, we stress that the position (and consequently the distance between) of the cascading modes remains unaffected by the change in the amplitude.

\subsection{Effect of the excitation wavenumber}

On Figures 4 and 5 the base wavenumber $k_{0}$ was fixed and taken from Table 1 . In this Section we describe the numerical experiments for another value of the parameter $k_{0}=5 \times 1.884$. The results of numerical simulations are reported on Figures 6 and 7 . Namely, on Figure 6 it is shown that the new exponent of the spectrum is much lower 

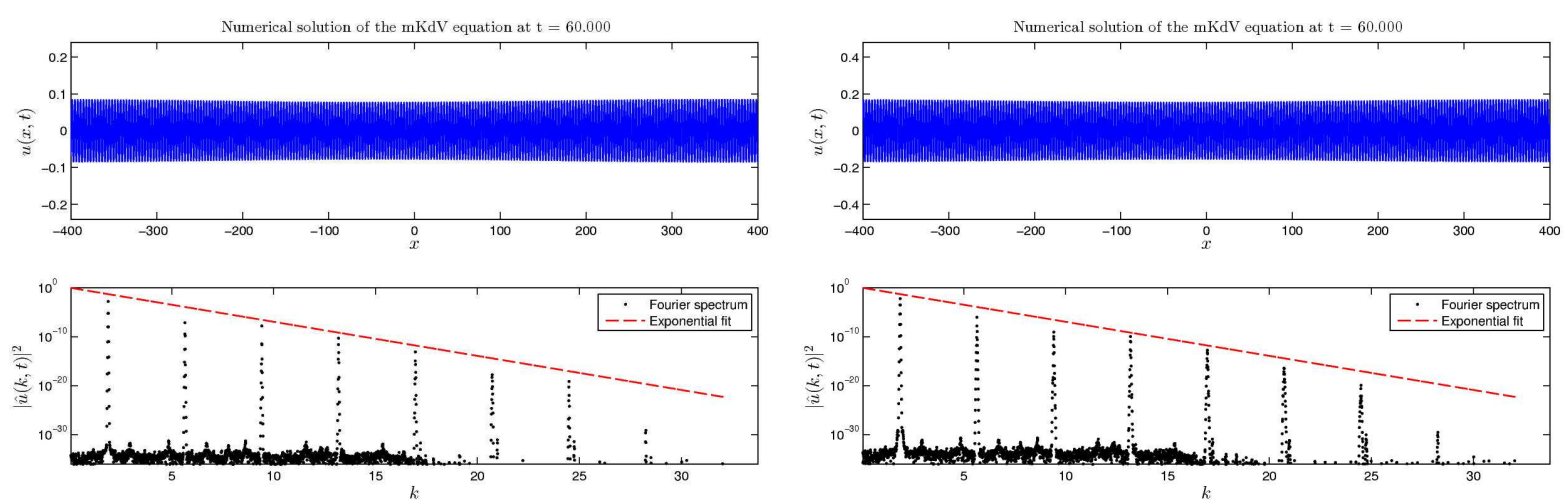

(a) $a=0.08, t=60$

(b) $a=0.16, t=60$
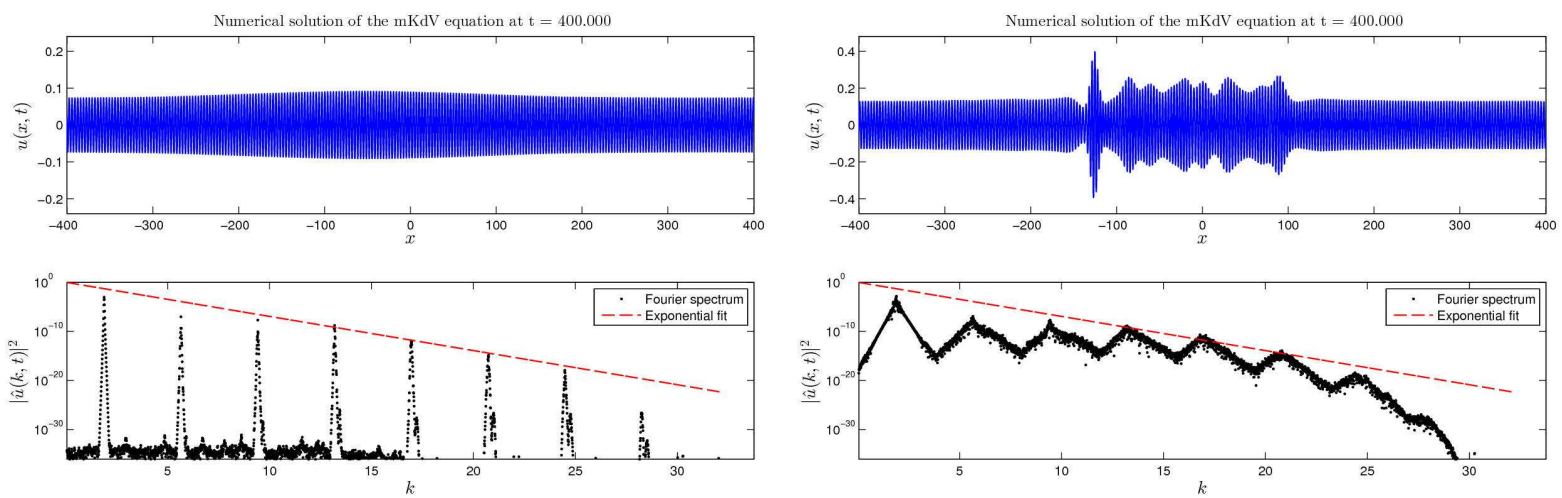

(c) $a=0.08, t=400$

(d) $a=0.16, t=400$
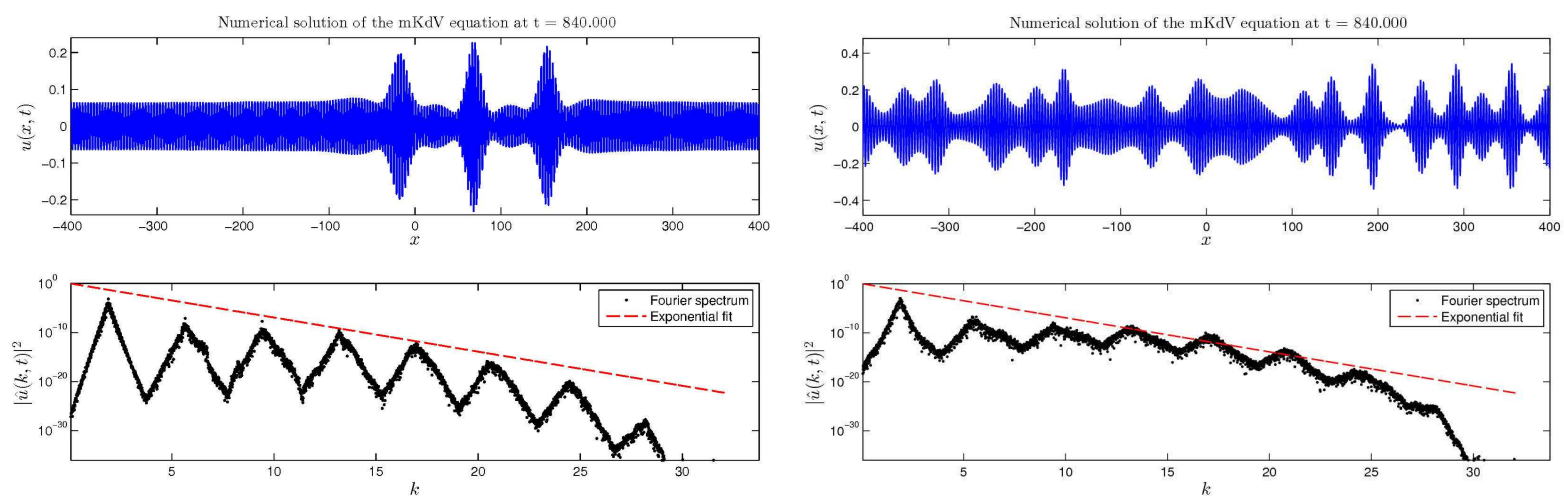

(e) $a=0.08, t=840$

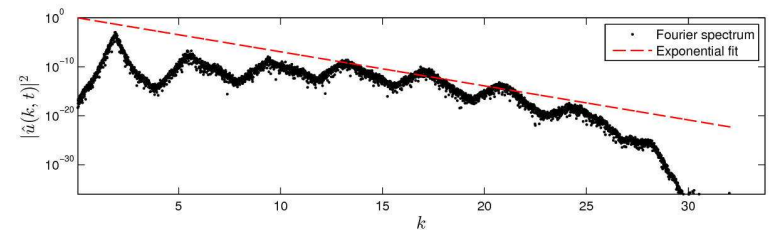

(f) $a=0.16, t=840$

Figure 4. Development of the $M I$ in the $m K d V$ equation for the initial wave amplitudes $a=0.08$ (left panel) and $2 a=0.16$ (right panel) and several simulation times.

than in the previous case. Moreover, if we increase the base wave amplitude $a$ for the new value of $k_{0}=9.42$, the spectrum shape remains constant, however we cannot affirm anymore that it is still exponential as it is hinted on Figure 7. The positions and energies of cascading modes for these two simulations are reported in Table 3. From these observation 

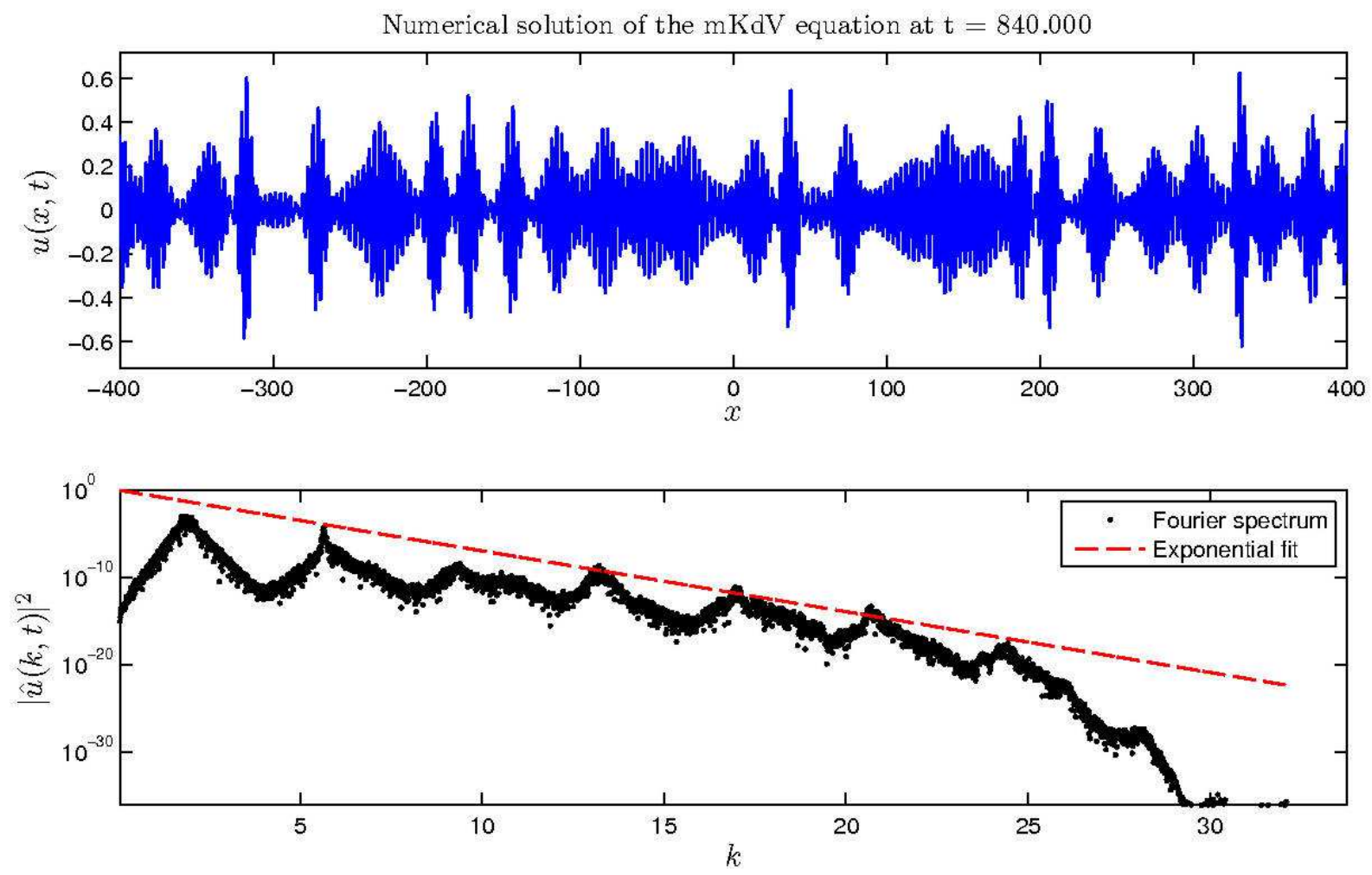

Figure 5. Development of the MI in the $m K d V$ equation for the initial wave amplitude $3 a=0.24$ at the final simulation time $T=840$.

\begin{tabular}{|c|c|c|c|c|c|c|}
\hline$a$ & 0.08 & & & & & \\
\hline$k$ & 9.428 & 28.26 & 47.11 & 65.94 & 84.8 & 103.6 \\
\hline$\varepsilon$ & $3.35 \times 10^{-4}$ & $2.83 \times 10^{-5}$ & $5.07 \times 10^{-6}$ & $6.35 \times 10^{-7}$ & $5.29 \times 10^{-10}$ & $1.79 \times 10^{-13}$ \\
\hline$a$ & 0.16 & & & & & \\
\hline$k$ & 9.396 & 28.27 & 47.09 & 65.92 & 84.77 & 103.6 \\
\hline $\mathcal{\varepsilon}$ & $1.04 \times 10^{-3}$ & $4.30 \times 10^{-5}$ & $1.18 \times 10^{-5}$ & $8.15 \times 10^{-8}$ & $3.52 \times 10^{-10}$ & $3.2 \times 10^{-14}$ \\
\hline
\end{tabular}

Table 3. Wavenumbers and energies of cascading modes for $a=0.08$ and $a=0.16$. The simulation snapshots are shown on Figure $7(a, b)$ correspondingly.

one can clearly see that the initial wave amplitude does not affect the structure of the $D$-cascade.

Another important consequence of the variation of the parameter $k_{0}$ is the distance between cascading modes in the Fourier space. More precisely, the distance increases with the increase in $k_{0}$. For example, our simulations show that the distance increases from $\Delta k \approx 3.81$ to 18.42 when $k_{0}$ goes from 1.884 to $5 \times 1.884=9.42$. 

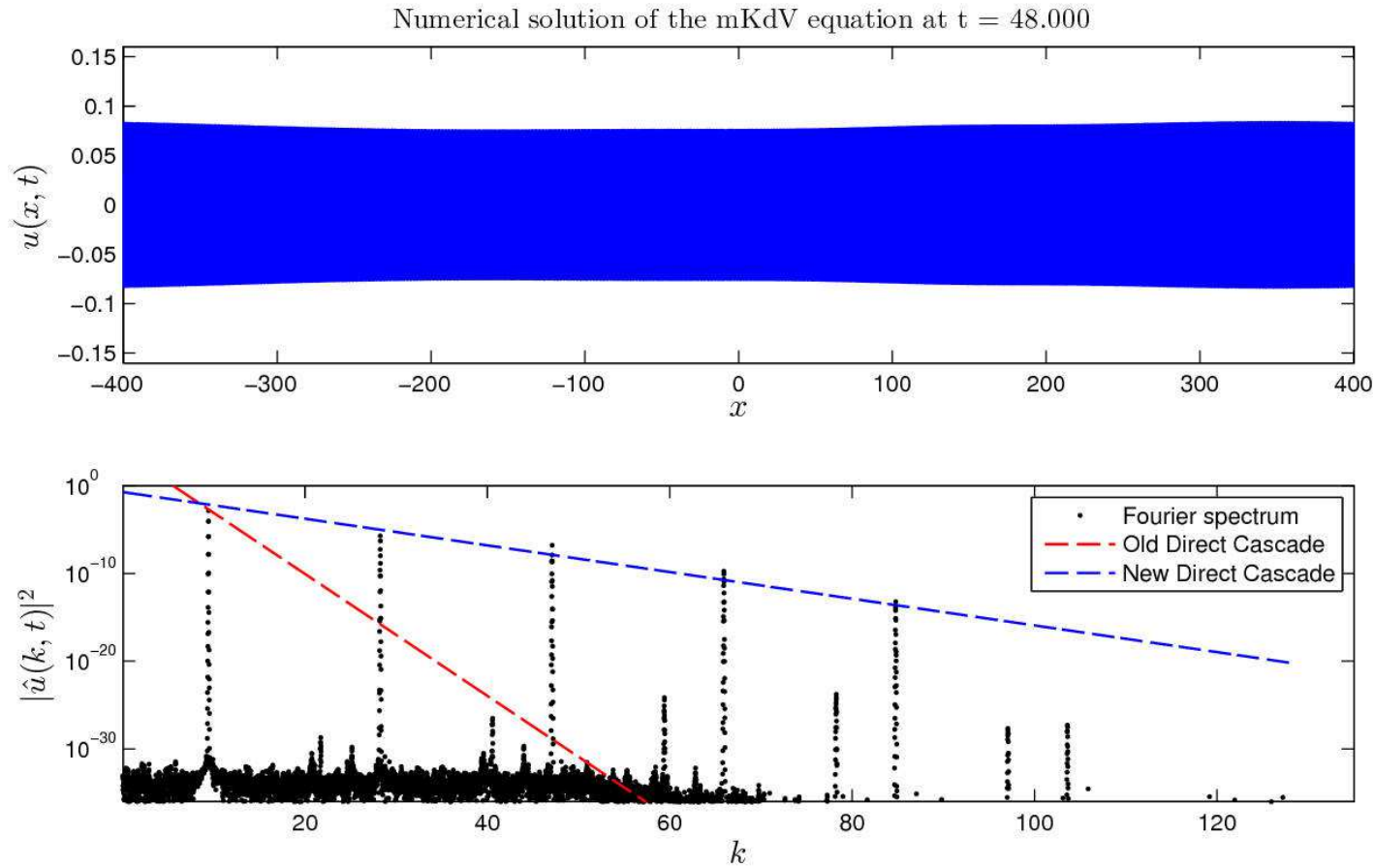

(a) $t=48$
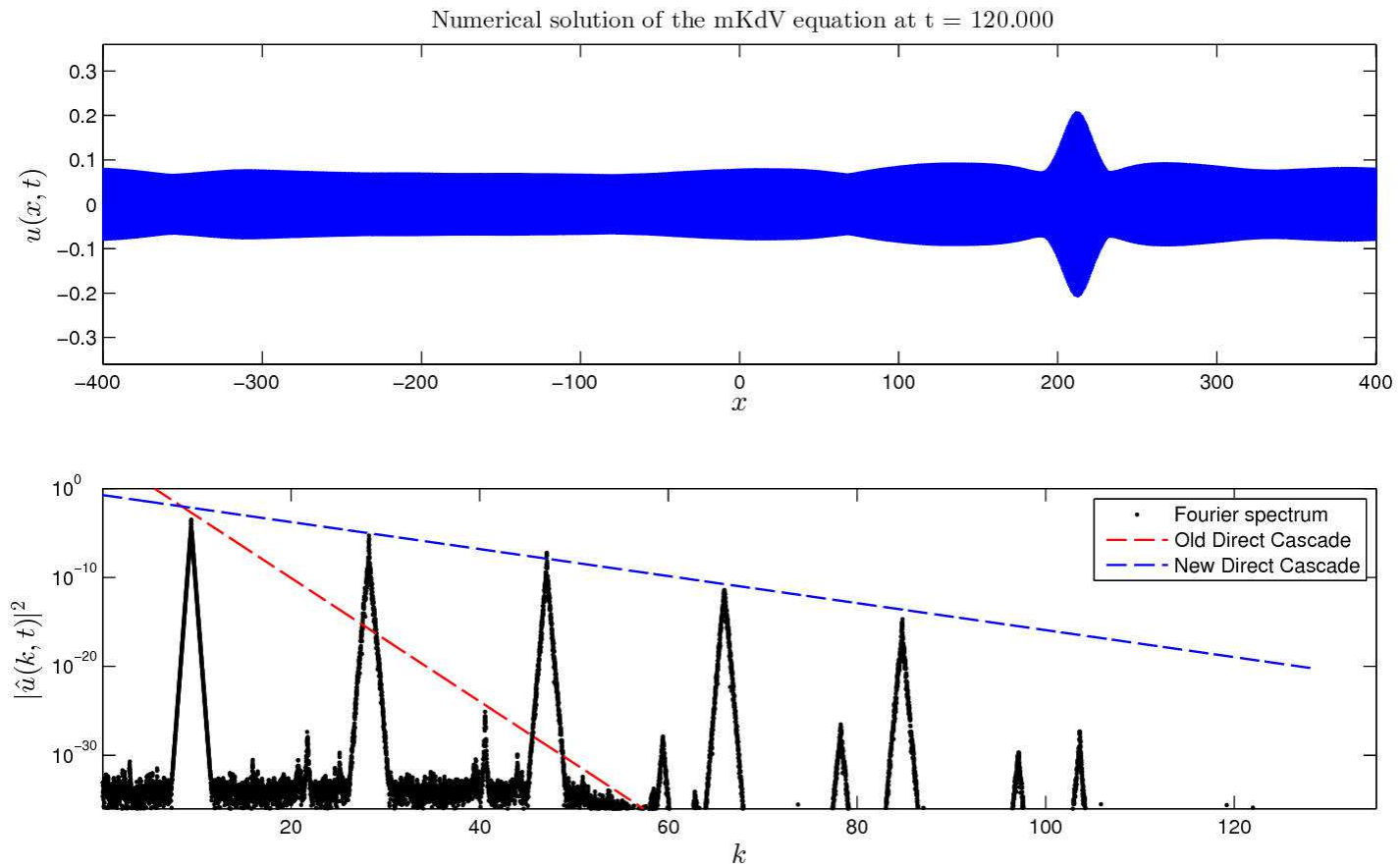

(b) $t=150$

Figure 6. MI simulation for $k_{0}=5 \times 1.884$, all the other parameters are the same as given in Table 1. The red dashed line on the bottom panels indicates the fit of the previous simulations for $k_{0}=1.884$. The blue dashed line shows the new fit. The magenta dashed line shows the direction of the eventual inverse cascade. 

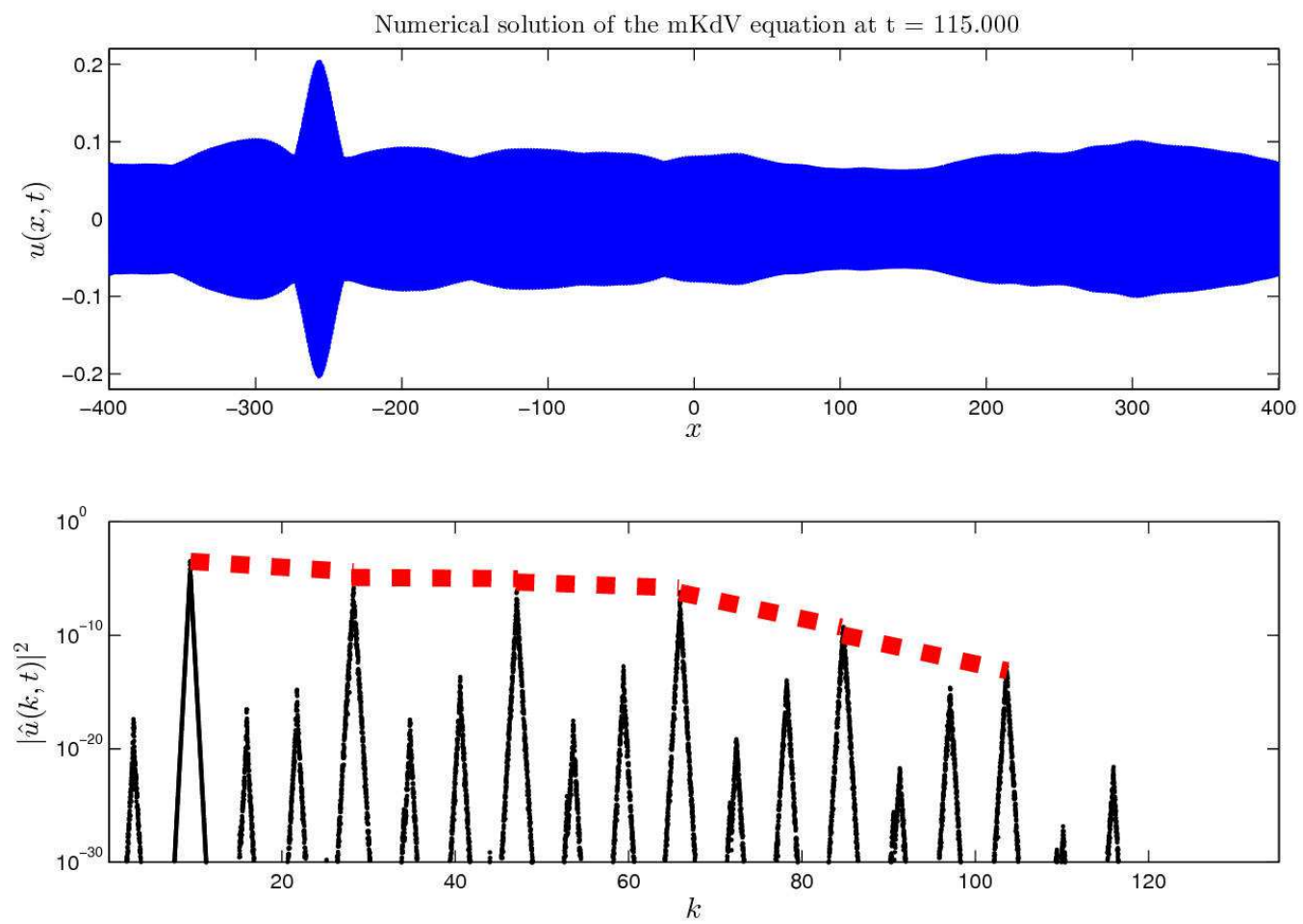

(a) $a=0.08, t=115$
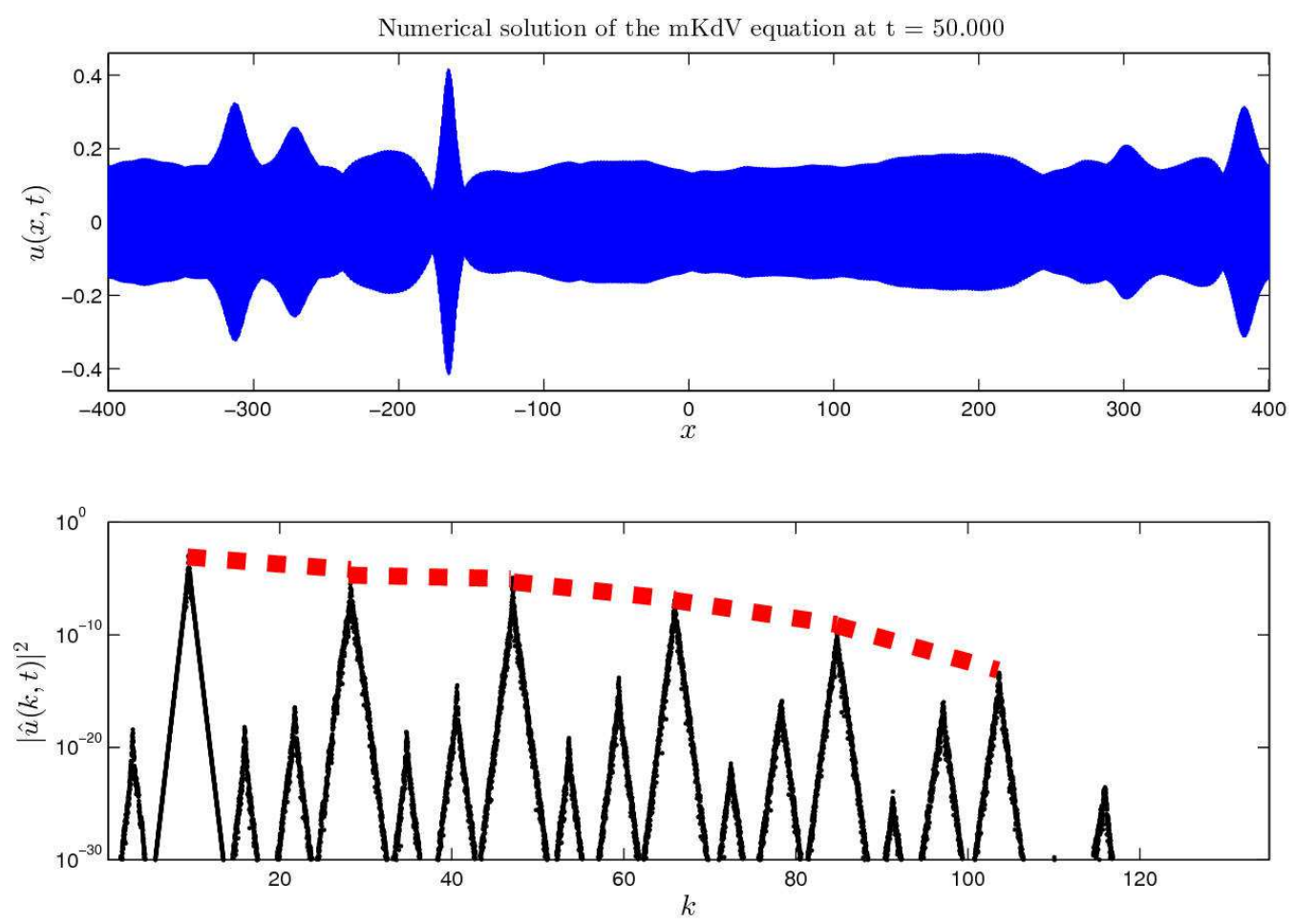

(b) $a=0.16, t=50$

Figure 7. MI simulation for $k_{0}=5 \times 1.884$, and two different values of the base wave amplitude $a$. The red bold dotted line corresponds to the energy spectrum shape observed in the numerical simulation. 


\subsection{Effect of the perturbation magnitude}

In the numerical simulations performed so far we have always taken the perturbation with magnitude $m=0.05$, which results in $5 \%$ amplitude modulation in terms of the base wave amplitude $a$. We tested various values of parameter $m$ in our numerical tests. Here we report on Figure 8 the most severe case of $m=0.5$ (i.e. $50 \%$ of the perturbation). Even in such an extreme case, the $D$-cascade is still present. However, we cannot state anymore with certitude that its shape is exponential. This questions will require more detailed investigations with even more resolved numerical simulations.

\section{Conclusions}

In the present study we have investigated the $D$-cascade formation in the framework of the $\mathrm{mKdV}$ equation. The main mechanism generating this cascade is the Modulational Instability (MI). One of the first theoretical studies devoted to the MI in the mKdV equation is [15], where the transformation from the mKdV to the NLS equation was highlighted. This transformation allows to find the conditions when the MI occurs in mKdV. Their theoretical study was illustrated with some numerical simulations showing the development of the MI in the physical space only. Since the $D$-model (or any other model) for energy transfer via the MI was not proposed yet at that time, the energy transport across the Fourier space was not in the focus. Consequently, the present study can be considered to be complementary since the main focus here is precisely on what is going on in the Fourier space.

In our numerical experiments we studied the nonlinear stage of the MI evolution aiming to observe the evolution of Fourier spectra additionally to the wave observation in the physical space, already reported in previous studies [15, 29]. The main findings can be briefly summarized in the following list:

- For a wide class of the initial conditions leading to the MI we clearly observe the formation of the direct (i.e. in the direction of increasing wavenumbers $k$ ) $D$-cascade. The ranges of parameters considered in this study are given here:

Amplitude: $a=0.01 \div 0.24$

Perturbation magnitude: $m=0.05 \div 0.5$

Base wavenumber: $k_{0}=1.8 \div 60.0$

Simulation time horizon: $T=40 \div 2000 \propto \mathcal{O}\left(\varepsilon^{-2}\right)$

Number of Fourier harmonics: $N=1024 \div 131072$ 

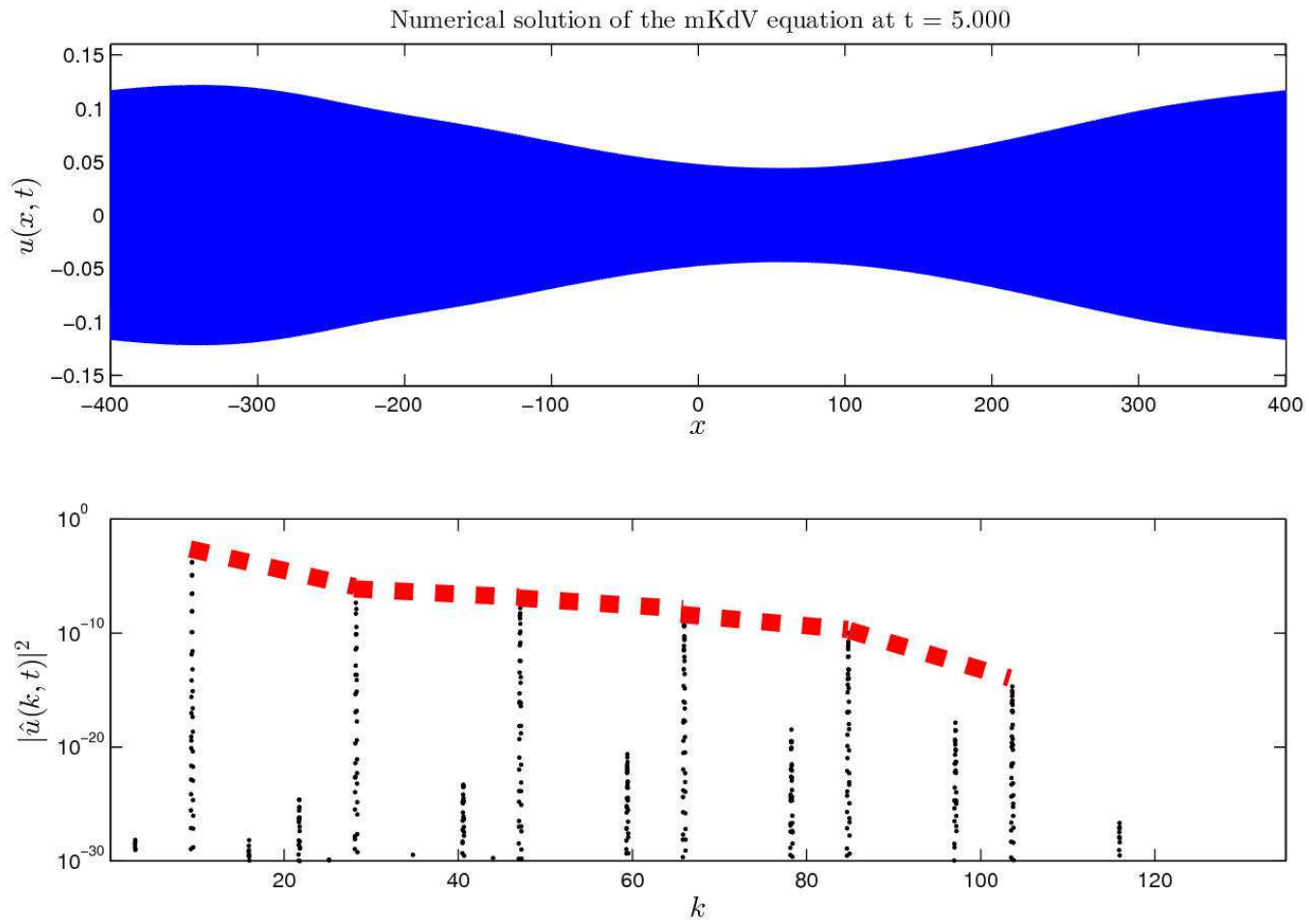

(a) $t=5$
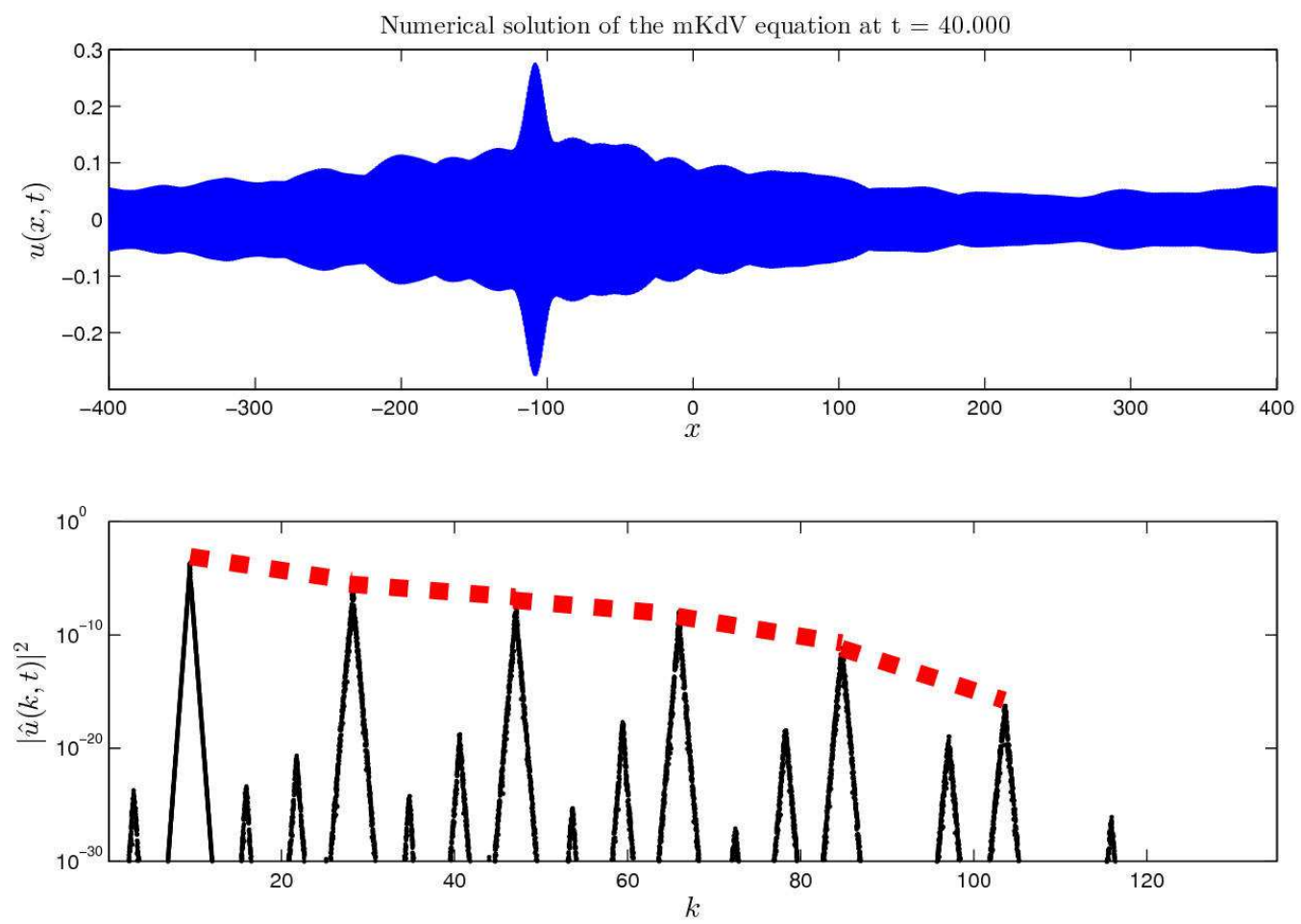

(b) $t=40$

Figure 8. MI simulation for $k_{0}=5 \times 1.884$, and the perturbation magnitude $m=10 \times 0.05=0.5$. The red bold dotted line corresponds to the energy spectrum shape observed in the numerical simulation. 
- In our numerical simulation the MI develops on the dynamical time scales of the order of $\mathcal{O}\left(\varepsilon^{-2}\right)$ which is in agreement with theoretical predictions [24].

- It is interesting to note that this time scale refers to the complete development of the MI in the physical space (see Figure $4(d, e)$ ). On the other hand one can see that the main structure of the $D$-cascade is already observable in Fourier spectra from the first instances of the dynamical evolution. Consequently, the development of the MI in the physical space corresponds to the spectral broadening of cascading modes. It is remarkable that their positions and energies are quasi-stationary.

- The observed $D$-cascade skeleton in the Fourier space is very robust and it has the exponential decay $\mathcal{E}_{k} \propto \exp (-\alpha \cdot k)$ (see Figures 4 and 5 ). The exponent $\alpha$ was found to be independent of the base wave amplitude $a$ for fixed values of other parameters.

- The increase of $k_{0}$ (the base wave wavenumber) has more drastic consequences on the spectrum shape. The agreement with the exponential spectrum $\varepsilon_{k} \propto$ $\exp (-\alpha \cdot k)$ is not satisfactory anymore. This highly nonlinear regime requires a more detailed investigation with higher resolutions in the Fourier space in order to obtain a longer cascade before making some conclusions about its shape (most of simulations presented in this study were already performed with $N=32768$ Fourier harmonics).

- We performed a series of numerical experiments for different values of the perturbation magnitude $m=0.05 \div 0.5$. We conclude that the main features of the $D$-cascade described above are preserved (see Figure 8 ). The choice of the perturbation influences the time scale on which the MI will develop in the physical space. Generally, higher magnitudes of the perturbation tend to accelerate this process.

\section{Acknowledgments}

The authors would like to thank Professors Mat JoHnson (University of Kansas) and Fritz Gesztesy (University of Missouri) for interesting discussions and most useful comments. This research has been supported by the Austrian Science Foundation (FWF) under projects P22943-N18 and P24671.

\section{References}

[1] M. J. Ablowitz and H. Segur. Solitons and the Inverse Scattering Transform. Society for Industrial \& Applied Mathematics, 1981. 5

[2] S. Y. Annenkov and V. I. Shrira. Role of non-resonant interactions in the evolution of nonlinear random water wave fields. J. Fluid Mech., 561:181-207, aug 2006. 4

[3] T. B. Benjamin and J. E. Feir. The disintegration of wavetrains in deep water. Part 1. J. Fluid Mech., 27(417), 1967. 4, 5, 6 
[4] J. P. Boyd. Chebyshev and Fourier Spectral Methods. New York, 2nd edition, 2000. 7

[5] J. C. Bronski, M. A. Johnson, and T. Kapitula. An index theorem for the stability of periodic travelling waves of Korteweg-de Vries type. Proc. R. Soc. Edinburgh Sect. A, 141(06):11411173, nov 2011. 8

[6] J. R. Cash and A. H. Karp. A variable order Runge-Kutta method for initial value problems with rapidly varying right-hand sides. ACM Transactions on Mathematical Software, 16(3):201-222, sep 1990. 7

[7] P. Denissenko, S. Lukaschuk, and S. Nazarenko. Gravity Wave Turbulence in a Laboratory Flume. Phys. Rev. Lett., 99(1):014501, jul 2007. 6

[8] H. R. Dullin, G. A. Gottwald, and D. D. Holm. On asymptotically equivalent shallow water wave equations. Phys. D, 190(1-2):1-14, mar 2004. 5

[9] D. Dutykh and E. Tobisch. Observation of the Inverse Energy Cascade in the modified Korteweg-de Vries Equation. EPL, 107:14001, 2014. 7

[10] K. B. Dysthe. Note on a modification to the nonlinear Schrödinger equation for application to deep water. Proc. R. Soc. Lond. A, 369:105-114, 1979. 4

[11] M. Frigo. A fast Fourier transform compiler. In Proc. 1999 ACM SIGPLAN Conf. on Programming Language Design and Implementation, volume 34, pages 169-180. ACM, may 1999. 7

[12] M. Frigo and S. G. Johnson. FFTW: An adaptive software architecture for the FFT. In Proc. 1998 IEEE Intl. Conf. Acoustics Speech and Signal Processing, volume 3, pages 1381-1384. IEEE, 1998.

[13] M. Frigo and S. G. Johnson. The Design and Implementation of FFTW3. Proceedings of the IEEE, 93(2):216-231, 2005. 7

[14] R. Grimshaw. Internal Solitary Waves. In R. Grimshaw, editor, Environmental Stratified Flows, pages 1-27. Springer US, 2002. 7

[15] R. Grimshaw, D. E. Pelinovsky, E. N. Pelinovsky, and T. Talipova. Wave group dynamics in weakly nonlinear long-wave models. Phys. D, 159(1-2):35-57, nov 2001. 5, 17

[16] R. Grimshaw, E. N. Pelinovsky, T. Talipova, M. S. Ruderman, and R. Erdelyi. ShortLived Large-Amplitude Pulses in the Nonlinear Long-Wave Model Described by the Modified Korteweg-De Vries Equation. Stud. Appl. Math., 114(2):189-210, feb 2005. 9

[17] E. Hairer and G. Wanner. Solving Ordinary Differential Equations II. Stiff and DifferentialAlgebraic Problems. Springer Series in Computational Mathematics, Vol. 14, 1996. 7

[18] M. Haragus and T. Kapitula. On the spectra of periodic waves for infinite-dimensional Hamiltonian systems. Phys. D, 237(20):2649-2671, oct 2008. 5

[19] S. J. Hogan. The fourth-order evolution equation for deep-water gravity-capillary waves. Proc. R. Soc. A, 402(1823):359-372, 1985. 4

[20] T. Y. Hou and R. Li. Computing nearly singular solutions using pseudo-spectral methods. J. Comp. Phys., 226(1):379-397, sep 2007. 7

[21] M. A. Johnson. Stability of Small Periodic Waves in Fractional KdV-Type Equations. SIAM J. Math. Anal., 45(5):3168-3193, oct 2013. 5

[22] E. Kartashova. Energy spectra of 2D gravity and capillary waves with narrow frequency band excitation. EPL, 97(3):30004, feb 2012. 4, 6

[23] E. Kartashova. Energy transport in weakly nonlinear wave systems with narrow frequency band excitation. Phys. Rev. E, 86(4):041129, oct 2012. 4

[24] E. Kartashova. Time scales and structures of wave interaction exemplified with water waves. EPL, 102(4):44005, may 2013. 4, 12, 19 
[25] A. N. Kolmogorov. The Local Structure of Turbulence in Incompressible Viscous Fluid for Very Large Reynolds Numbers. Proc. R. Soc. Lond. A, 434(1890):9-13, jul 1991. 4

[26] R. M. Miura, C. S. Gardner, and M. D. Kruskal. Korteweg-de Vries Equation and Generalizations. II. Existence of Conservation Laws and Constants of Motion. Journal of Mathematical Physics, 9(8):1204, 1968. 8

[27] A. C. Newell and B. Rumpf. Wave Turbulence. Ann. Rev. Fluid Mech., 43(1):59-78, jan 2011. 4

[28] H. Ono. Algebraic Soliton of the Modified Korteweg-de Vries Equation. J. Phys. Soc. Jpn., 41(5):1817-1818, nov 1976. 8

[29] M. S. Ruderman, T. Talipova, and E. Pelinovsky. Dynamics of modulationally unstable ionacoustic wavepackets in plasmas with negative ions. J. Plasma Phys., 74(05):639-656, apr 2008. 17

[30] H. Schamel. A modified Korteweg-de Vries equation for ion acoustic wavess due to resonant electrons. J. Plasma Phys., 9(03):377-387, mar 1973. 5

[31] E. Tobisch. Energy spectrum of ensemble of weakly nonlinear gravity-capillary waves on a fluid surface. JETP, 119(2):359-365, 2014. 4

[32] L. N. Trefethen. Spectral methods in MatLab. Society for Industrial and Applied Mathematics, Philadelphia, PA, USA, 2000. 7

[33] M. P. Tulin and T. Waseda. Laboratory observations of wave group evolution, including breaking effects. J. Fluid Mech., 378:197-232, jan 1999. 6

[34] G. B. Whitham. Variational Methods and Applications to Water Waves. Proc. R. Soc. Lond. A, 299(1456):6-25, jun 1967. 5

[35] H. Xia, M. Shats, and H. Punzmann. Modulation instability and capillary wave turbulence. EPL, 91(1):14002, jul 2010. 6

[36] N. J. Zabusky and C. C. J. Galvin. Shallow Water Waves, the Korteweg-de Vries Equation and Solitons. J. Fluid Mech., 47:811-824, 1971. 5

[37] V. E. Zakharov, V. S. Lvov, and G. Falkovich. Kolmogorov Spectra of Turbulence I. Wave Turbulence. Series in Nonlinear Dynamics, Springer-Verlag, Berlin, 1992. 4

LAma, UmR 5127 CNRS, Université de Savoie, Campus Scientifique, 73376 Le BourgetDu-Lac Cedex, France

E-mail address: Denys.Dutykh@univ-savoie.fr

$U R L:$ http://www.denys-dutykh.com/

Institute for Analysis, Johannes Kepler University, Linz, Austria

E-mail address: Elena.Tobisch@jku.at

URL: http://www.dynamics-approx.jku.at/lena/ 